\title{
Assessing equity in transport accessibility to work and study: The Bogotá region
}

Luis A.Guzman, Departamento de Ingeniería Civil y Ambiental, Universidad de los Andes, Bogotá, Colombia. Email: la.guzman@uniandes.edu.co

Daniel Oviedo, Development Planning Unit, University College London, London, UK. Email: daniel.oviedo@ucl.ac.uk

Carlos Rivera, Departamento de Ingeniería Civil y Ambiental, Universidad de los Andes, Bogotá, Colombia. Email: ci.rivera52@uniandes.edu.co

\begin{abstract}
This research was aimed at exploring levels of equity in accessibility to employment and education in the city-region of Bogotá, Colombia's capital city. Building on consolidated methodologies for the assessment of potential accessibility, we estimate accessibility indicators at the zone level, evaluate how potential accessibility varies among income groups, and present evidence related to transport mode, in order to analyze social and spatial inequalities produced by the distribution of accessibility to employment and education activities. The research incorporates a method to evaluate how accessibility varies among zones according to average income and mode of transport in order to produce evidence-based arguments that can inform transport policy in the city-region of Bogotá, and other similar contexts in the Global South. Our results show strong distributional effects of the socio-spatial and economic structure of the city-region, its transport infrastructure and services, and the effect of current transport and land-use policies for citizens of different income groups. The tools and empirical evidence in this research seek to contribute to informed policy development in Latin America and other developing contexts, and feeding current debates on the role of accessibility in addressing social and spatial inequalities stemming from urban mobility.
\end{abstract}

Keywords: access inequalities; potential accessibility; commuting access; Bogotá

\section{Introduction}

Inequalities in the distribution of costs and benefits of transport-related infrastructure and services can contribute to the production and reproduction of social inequalities and levels of exclusion in cities (Manderscheid, 2009). Low-income and other socially vulnerable populations are often forced to endure the greatest negative effects of poorquality transport, including longer journey times, and increased exposure to pollution and risk of traffic accidents (Titheridge et al., 2014). Transport and mobility plans and regulations in major cities in Latin America tend to incorporate increased social inclusion and equity as central objectives in their discourse (Scholl et al., 2016). However, planning and development tools for the analysis and evaluation of projects and conditions of transport used in practice often do not incorporate specific or rigorous indicators that permit measuring their contribution to levels of accessibility to the opportunities the city offers, and their social implications (Geurs and van Wee, 2004;Lucas and Jones, 2012;van Wee, 2016). This is partly a result of traditional planning methods for urban transport that focused on demand and efficiency, and which do not explicitly consider social or spatial equity (Keeling, 2008a;Keeling, 2008b). There is a need for further empirical evidence demonstrating the relationship between the provision of transport, social transport needs, and the differentiated effects of the interactions between transport, land use and individual characteristics, particularly for low income and other socially vulnerable groups (Geurs and van Wee, 2004).

In Colombia, there has been a recent increase in the scientific literature that explores the concept of accessibility, which is understood in this study as the ease to reach opportunities available throughout a metropolitan space from a given origin location 
using available transport options. Studies in different cities in Colombia provide examples of various methodological approaches to the study of accessibility (see for example Bocarejo and Oviedo (2012); Delmelle and Casas (2012); and Escobar and Garcia (2012)). Many studies in the local context focus on the relationships between accessibility with land-uses and prices, and the effect of recent investments in urban transport on both spatial and social inequalities (Bocarejo et al., 2014;Grindlay et al., 2012; Munoz-Raskin, 2010). Such research has contributed to the consolidation of accessibility measures for policy analysis, transport conditions and practices that are applicable in Colombian cities, as well as cities that have similar characteristics elsewhere. However, new research exploring further advances in methodologies and evidence, and using more sophisticated data to explore social inequalities stemming from urban transport, can add to current debates and practice related to the measuring and planning of transport accessibility in cities of Colombia and other countries of the Global South.

This study contributes to debates regarding transport accessibility and social equity by providing new evidence and methodological elements from the study of daily mobility in what we term the 'Bogotá metropolitan region' $\left(\mathrm{BMR}^{1}\right)$. Our study provides evidence at the metropolitan level, which has not yet been fully explored as previous research has largely focused on the city of Bogotá despite clear interdependences between the city and its surrounding municipalities (Oviedo and Dávila, 2016). We also incorporate differentiated analysis by modes of transport (car, regular public transport (bus) and BRT - Transmilenio (TM)), in an attempt to identify the contribution of different transport alternatives to the accessibility of various social groups and settlements throughout the metropolitan region. The research makes a differentiation between the TM, Colombia's first Bus Rapid Public transport system (Guzman et al., 2016; Hidalgo and Sandoval, 2004), and traditional buses as they have very different operational characteristics. The $\mathrm{TM}$ has become one of the most common topics of research concerning urban transport in Colombia, and it has been globally recognized as good practice in sustainable mobility (Gilbert, 2008;Hidalgo and Sandoval, 2004). The system operates under a trunk-feeder structure with stations located in the center of the road, has exclusive lanes for trunk buses, payment kiosks at the station and large-capacity vehicles. Only four years after the implementation of TM the average travel times in the city decreased by $32 \%$, accidents in the corridors where the system operates were reduced by $90 \%$, and air pollution due to emissions of particulate matter decreased by $9 \%$. Therefore, it is considered relevant to estimate the extent to which the system is contributing to accessibility in the areas it currently covers.

Our research builds on widely discussed methodologies in the international literature such as potential accessibility (Geurs and Ritsema van Eck, 2001;Geurs and van Wee, 2004;Preston and Rajé, 2007), and approaches to the study of inequalities applicable to transport (Delbosc and Currie, 2011b;Grengs, 2015; Lucas et al., 2016). This study assesses levels of accessibility throughout the BMR using information from an integrated transport and land-use model and secondary information from the largest up-to-date household transport survey in the region. Calculations consider the generalized cost of travel between the zones of origin and opportunities at different destinations. Opportunities are measured as the sum of jobs and education positions. Thus, accessibility in this case is defined as the potential extent to which the transport system and spatial distribution of opportunities allow individuals to reach work and education

\footnotetext{
${ }^{1}$ The BMR is an unofficial definition of the city-region of Bogotá based on functional interactions between the city and surrounding municipalities. There is no current metropolitan authority defined for the region despite large flows of people and goods, and transport and connectivity networks and services facilitating travel between Bogotá and the municipalities included in the BMR.
} 
using a given mode of transport at a zonal level. This definition encompasses dimensions of both transport (generalized cost) and land-use (spatial distribution of activities).

\section{Transport accessibility and social equity}

Growing concern about the social consequences of mobility has led to a visible increase in research exploring the links between transport, poverty, welfare and sustainability (Hine, 2009; Jones and Lucas, 2012). Transport provides the means to access essential opportunities for participation in society, such as employment, education, trade and social activities, which therefore links a lack of mobility to social disadvantage and exclusion (Lucas, 2012). Accessibility can be defined and calculated in different ways, with various implications. These include well-known definitions such as: the potential of opportunities available for an individual (or groups) located at a certain location for interaction (Hansen, 1959); the available opportunities that allow individuals to participate in one (or more) particular activities, considering travel purposes or larger activity-based travel (Ben-Akiva et al., 2006); or the capacity of a location to reach and be reached from different locations through the transport system (Dalvi and Martin, 1976).

Geurs and Van Wee (2004) identify four main components in the concept of accessibility: (i) land-use, which refers to the quantity, quality and distribution in space of opportunities such as jobs, shops, healthcare and, social and recreational facilities at destination locations, and the demand for opportunities at origin locations; (ii) transport, which accounts for the features of the transport system expressed in terms of the (dis)utility for an individual to travel between origins and destinations using a given mode of transport; (iii) time, which reflects time constraints related to both availability of opportunities during the day, and the availability of time for individuals to make use of such opportunities; and, (iv) individual, which reflects the needs, abilities and opportunities of individuals that can influence levels of access to transport and their ability to participate in opportunities. The interactions between the components outlined above produce differentiated levels of accessibility by mode, location, social groups and activity. The focus on the different components of accessibility has led to various indicators and methodologies for its measurement, which include infrastructure-based measures, location-based measures and individual-based indicators as the dominant approaches, with more recent developments using mixed approaches to study the interrelations between different components (van Wee et al., 2013).

A clear example of the influence of transport on access to opportunities, and one of the most recurrent analyses in the international literature, is access to employment (Alam, 2009;Hess, 2005;Kawabata, 2003;Korsu and Wenglenski, 2010;Levinson, 1998;Sanchez et al., 2003). Transport was found to be an explanatory factor of great importance in the successful transition from education to employment (Wachs, 2010). As argued by Titheridge et al., (2014), there is a three-way dynamic relationship between jobs, housing and transport where access to employment is framed by: (i) the distance between housing and jobs; (ii) the availability of transport and its associated costs; and (iii) the availability of disposable income. The combination of such factors enables individuals to access transport, maintain mobility, and retain employment. In addition, low-income populations suffer from measurable deficits: nutrition, health, education, housing and job opportunities, which are invariably correlated with deficits in mobility (Titheridge et al., 2014).

Defining the relevance of transport and its impacts on accessibility is a relevant step toward more informed urban transport research. Arguably, the next step is to identify the distribution of accessibility between different social groups and geographical areas (Lucas et al., 2016). This is important because traditional land-use and transport policies generally overlook distributional effects, and how they affect the travel capacity of populations with different social identities, levels of income, or various skill and ability 
levles, which are distributed throughout urban areas. On the one hand, analysis of accessibility equity is relevant as one of the main objectives of transport policy is to provide equitable transport for all inhabitants of cities and metropolitan regions, in particular the poor and the socially disadvantaged. On the other hand, it is relevant to identify the distribution of accessibility not only from a transport perspective, but also considering the effect of land-use on travel behavior, in order to inform land-use policies and regulation in relation to their potential implications regarding the ability of different populations to access and make use of economic and social opportunities (van Wee and Handy, 2016). Different approaches can be found in the literature regarding the definition of equity in transport. Horizontal equity (Litman, 2014), requires that resources be allocated evenly to groups (or individuals), unless subsidies are specifically defined. As argued by Delbosc and Currie (2011a), this approach can be related to the principles of 'mass transit', which is aimed at maximizing the number of people being transported in an efficient and sustainable way. Vertical equity implies that disadvantaged groups (or individuals) must be identified in urban transport planning in order to design specific policies in their favor, in order to improve their current conditions (Litman, 2014). This is a more 'socially-focused' approach, where those with greater transport needs are targeted by policies aimed at increasing their access to both transport and opportunities (Deakin, 2007).

Different examples can be found in the international literature regarding the relationship between accessibility and equity, which stress the relevance of equity in the formulation and evaluation of public policies as outlined above (Delbosc and Currie, 2011a; Lucas et al., 2016;van Wee and Handy, 2016;Wu and Hine, 2003). Lucas et al., (2016) suggested that mainstream approaches to policy evaluation, such as cost-benefit or multi-criteria analysis do not fully address equity effects and risks the double counting of policy benefits, respectively. Recent transport research has incorporated the use of Lorenz curves and Gini indexes as scale-independent indicators for assessing accessibility and vertical equity in a holistic way (Delbosc and Currie, 2011a;Grengs, 2015;Karlström and Franklin, 2009;:Lucas et al., 2016). Such indicators are argued to be easy to interpret and to explain to policy and decision makers. Examples of these studies included the study by Delbosc and Currie (2011a), where the equity of the transit system in Melbourne, Australia was evaluated; and the research by Karlström and Franklin (2009), which studied the equity effects of the congestion pricing system in Stockholm. In addition, studies such as Wu and Hine (2003) and Grengs (2015), although they did not apply Lorenz curves, calculated accessibility for different social groups focusing on equity.

\section{Components of accessibility in the BMR}

Bogotá, one of the densest cities in the world in 2015 with 8.0 million people and an urban area of approximately $414 \mathrm{~km}^{2}$ of urban area, alongside its 12 surrounding municipalities, forms the largest urban conurbation in Colombia. Together, the most important neighboring municipalities (Soacha, Cajicá, Chía, Cota, Facatativá, Funza, La Calera, Madrid, Mosquera, Sopó, Tocancipá and Zipaquirá), represent an additional population of 1.2 million people in an area of $1,194 \mathrm{~km}^{2}$.

The concept of the metropolitan area in the Bogotá region is one that has gained increasing relevance in recent years. The governments of Bogotá and the municipalities, with support from the national government, have attempted, in different ways, to constitute a metropolitan authority for land-use and spatial planning (Acosta, 2010). However, an agreement towards the creation of such a metropolitan authority has not yet been possible, which makes the BMR the largest unofficial metropolitan region in Colombia (Guzman et al., 2017). Despite the lack of a comprehensive authority for coordinated planning and development, evidence suggests increasing economic and social interdependence between the municipalities and Bogotá (Acosta, 2010; Cámara de Comercio de Bogotá, 2015). According to data from the Mobility Survey and the 
Chamber of Commerce of Bogotá, there are large volumes of passengers travelling to Bogotá from nearby municipalities and vice versa, as well as an increase in regional economic activity in recent years (see SDM, 2011 and CCB, 2015).

The city of Bogotá is divided into 112 urban "zonal planning units" (UPZ), which are territorial units for planning urban development at the local level and for defining landuse and urban functions. Analysis of accessibility in Bogotá was conducted by UPZ. Considering the size of most surrounding municipalities, each municipality was analyzed as a unique zone, with the exception of Soacha, which is the largest municipality after Bogotá in the BMR (with 511,000 inhabitants in 2015), and was subsequently divided into four zones. As a result, the zoning of the BMR for the analysis in this paper included 127 zones. Although some of the municipalities in the BMR are farther from Bogotá and thus are not part of a continuous urban fabric (see for example Zipaquirá, Facatativá and Sopó -Figure 1-), large employment demand in these municipalities leads to travel to Bogotá and other municipalities in the region for work. In this regard, accessibility results for the municipalities can evidence spatial and social inequalities and inform debates regarding the relevance of integrated planning in the BMR. The main source of information for this research was the 2011 Mobility Survey for the city of Bogotá and its surrounding municipalities (SDM, 2011). The survey provides detailed data of sociodemographic and socioeconomic characteristics, travel patterns and preferences from a sample of 14,882 households distributed throughout Bogotá and the surrounding municipalities (ibid). This section summarizes the main characteristics of the BMR in relation to the main components of accessibility outlined in Section 2, thus providing the basis for the accessibility and equity analysis in the paper.

\subsection{Land use: distribution of activities and housing}

Distribution of the population in the BMR shows significant differences throughout the territory. Centralization of economic activities in the expanded city center, land regulations and housing market dynamics have made housing close to major centers of employment unaffordable for the poorest population. This has historically led this segment of the population to settle in informal settlements at the outskirts of Bogotá, particularly in the southern end of the city. In addition, an increase in the middle class and an increase in demand for large plots for high-end housing by higher-income groups has led to rapid housing development and suburbanization in western and northern municipalities (Thibert and Osorio, 2014). This has produced a complex conurbation process between Bogotá and some of its closest neighbors. As a result of these dynamics, settlements of low-income households in the BMR have very high densities in comparison with higher income areas, although even the latter present considerable densities according to international standards. The densest areas in the BMR are located mainly in the southern and western edges of the city. The poorest neighborhoods, which are also largely informal, can reach average values up to 56,000 inhabitants $/ \mathrm{km}^{2}$. By contrast, most of the central and northern sectors of the city are characterized by higher built-up areas, lower population densities, larger economic activity, and the highest concentration of employment in the region. These areas have densities between 6,000 and 15,000 inhabitants $/ \mathrm{km}^{2}$ (see Figure 1).

Concentration of employment and education opportunities in the BMR in the city center and other specific areas of Bogotá have caused major problems of inequality in terms of access to these activities, particularly affecting low-income groups (Bocarejo and Oviedo, 2012). A large share of income-generating and educational opportunities is concentrated along main transport corridors, especially in the north-central side of the city, where most high-income households are located (see Figure 1). Almost $44 \%$ of jobs in Bogotá are located in the large central core (12\% of the urban area). Despite some level of concentration of employment and education opportunities in the municipalities, in most cases these are related to specific economic sectors or levels of education (i.e., high-end private primary and secondary schools), which are insufficient for the local 
population both in terms of quantity and mismatch with individual characteristics such as education and income. Figure 1 shows the combined distribution of opportunities. However, it is relevant to clarify that despite a concentration of higher education opportunities near the city center, supply of primary and secondary education centers is more widely distributed with a greater supply in southern and north-western neighborhoods. This distribution is likely to improve accessibility in lower and middle-low income zones.
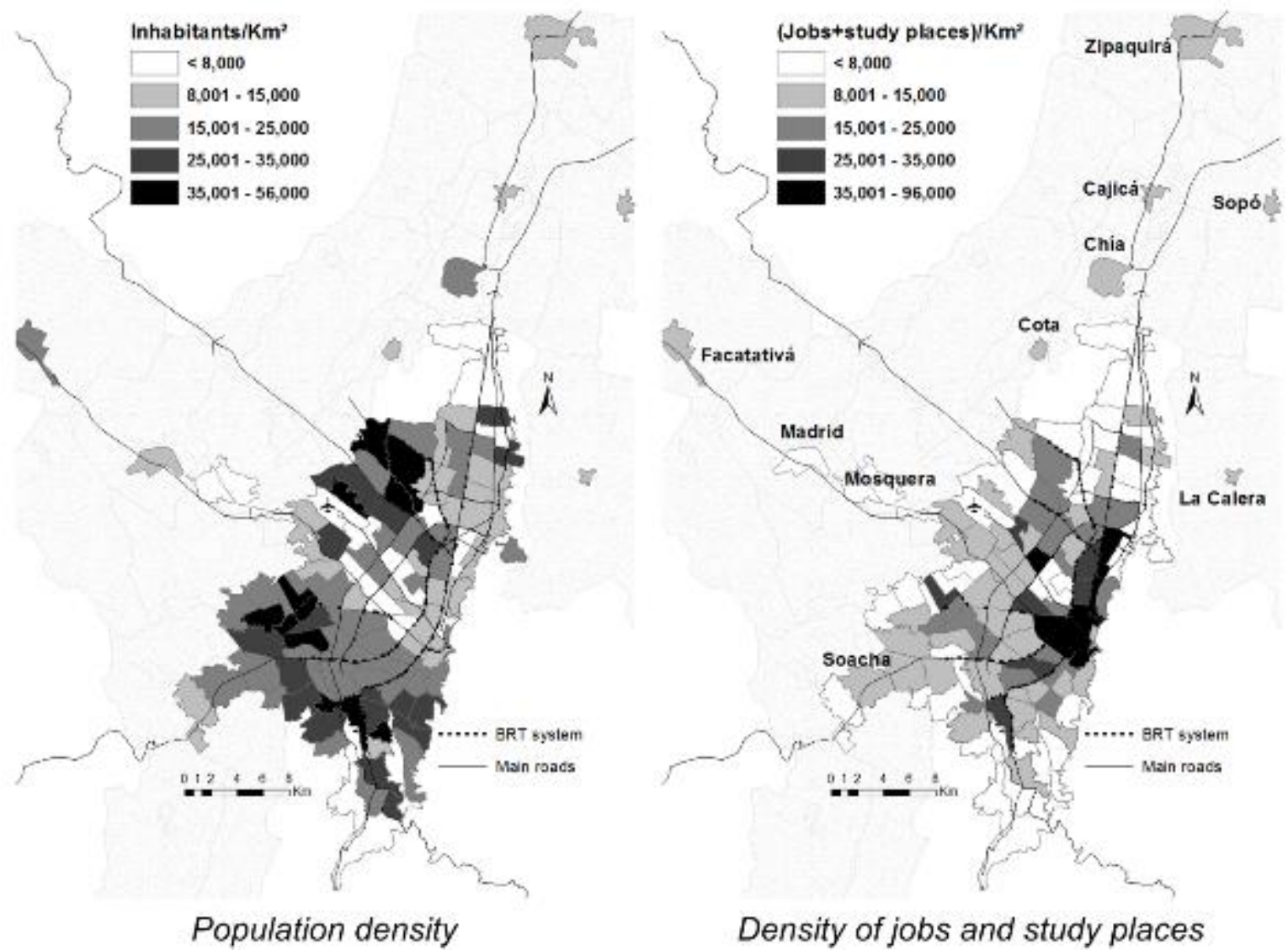

Figure 1. Distribution of population, jobs and study places

Source: Own elaboration based on data from Bogotá's Urban Planning Department

\subsection{Individual characteristics: socioeconomic features of the population}

Data for 2015 estimated the population of the BMR to be $9,205,125$ inhabitants, $68 \%$ of which was aged between 15 and 64 years, $26 \%$ aged at 14 years or less, and $6 \%$ over 65 years. Gender distribution in 2011 was $53 \%$ female and $47 \%$ male.

The BMR plays a central role in the national economy of Colombia, producing $24 \%$ of national Gross Domestic Product (CCB, 2015). Unemployment rate in Bogotá during the first trimester of 2015 was $8.6 \%$, which shows better conditions in relation to the national average of $9.5 \%$. Employment in the BMR was estimated at 4 million jobs in 2015 (CCB, 2015), although levels of informality in employment are estimated to be between 40 and $50 \%$.

Higher economic performance in the BMR has also led to meaningful reductions in economic poverty, with indices of poverty and extreme poverty below the average of other urban regions in the country. The poverty index of Bogotá for 2013 was 10.3\%, while the national average was $30.6 \%$. Similarly, the extreme poverty index was $1.6 \%$, far below the national average of $9.1 \%$ (DANE, 2013b). Education coverage indicators for the BMR are also more positive than the average of other cities in the country (DANE, 2013a). Coverage of primary and secondary education was nearly $86 \%$, while the 
national average was $75 \%$ in 2013 . Regarding higher education, coverage was $38 \%$ while the average for the rest of the country was $20 \%$ (ibid).

Among other variables, the Bogotá Mobility Survey collected data of household income expressed in terms of income brackets. Building on a frequency analysis of the data in the sample of the survey, we assigned a population weighted average household income range to each UPZ and municipality. Median income in the BMR according to this analysis is $1,583,700 \mathrm{COP} / \mathrm{month}$ (USD 830 approx.). For the purpose of this study we categorize income levels in three groups: low-income zones, whose average income is below the $50^{\text {th }}$ percentile (earning less than 1.17 million COP/month); middle-income zones, between the $50^{\text {th }}$ and $90^{\text {th }}$ percentiles (incomes between 1.17 million COP/month and 3,070,000 COP/month); and high-income zones, whose income is above the $90^{\text {th }}$ percentile. The spatial distribution of median income by zone is shown in Figure 2. Bogotá's surrounding municipalities are located below the $60^{\text {th }}$ percentile of the median income in the BMR. In this study, with the exception of Cota and Mosquera, the municipalities were classified as low income according to the data of the Bogotá Mobility Survey. Figure 2 (left) shows that lower-income households locate predominantly on the south and west sides of the city, including neighboring municipalities, away from areas with a higher density of opportunities for work and study (Figure 1, right). The effects of such a spatial mismatch include, among others, large travel times regardless of the transport mode used by the population.

\subsection{Transport: socially, economically and spatially differentiated costs}

Based on the above income levels, we found significant differences in travel costs between different parts of the region (Figure 2, travel times). Travel times were obtained directly from the sample of the Bogotá Mobility Survey and averaged at the zone level. Average travel time in the municipalities is $40 \%$ higher than in the city. As reflected by these indicators, a person with access to a car in a low-income zone spends $46 \%$ more time travelling than a car user in a high-income area. In addition, the travel time of people in lower income groups to their place of work and/or study (67 $\mathrm{min}$ ) are on average $22 \%$ longer than the middle-income group (55 min) and 40\% more than high-income zones (48 min). The inclusion of the municipalities in the analysis of the paper may arguably have an influence in the average travel times and costs involved in the accessibility estimations as a result of the greater distances to the city center. However, social and spatial segregation of the metropolitan region, distribution of population by levels of income throughout the BMR, and the considerable volume of passengers traveling between the municipalities and Bogotá every day (around 271,000), suggest large interdependence between municipalities regarding employment and education opportunities. Therefore, the inclusion of the municipalities as part of the analysis seeks to provide insights on travel dynamics in the region and add evidence to the discussions regarding the need for integrated (transport) planning in the BMR. 


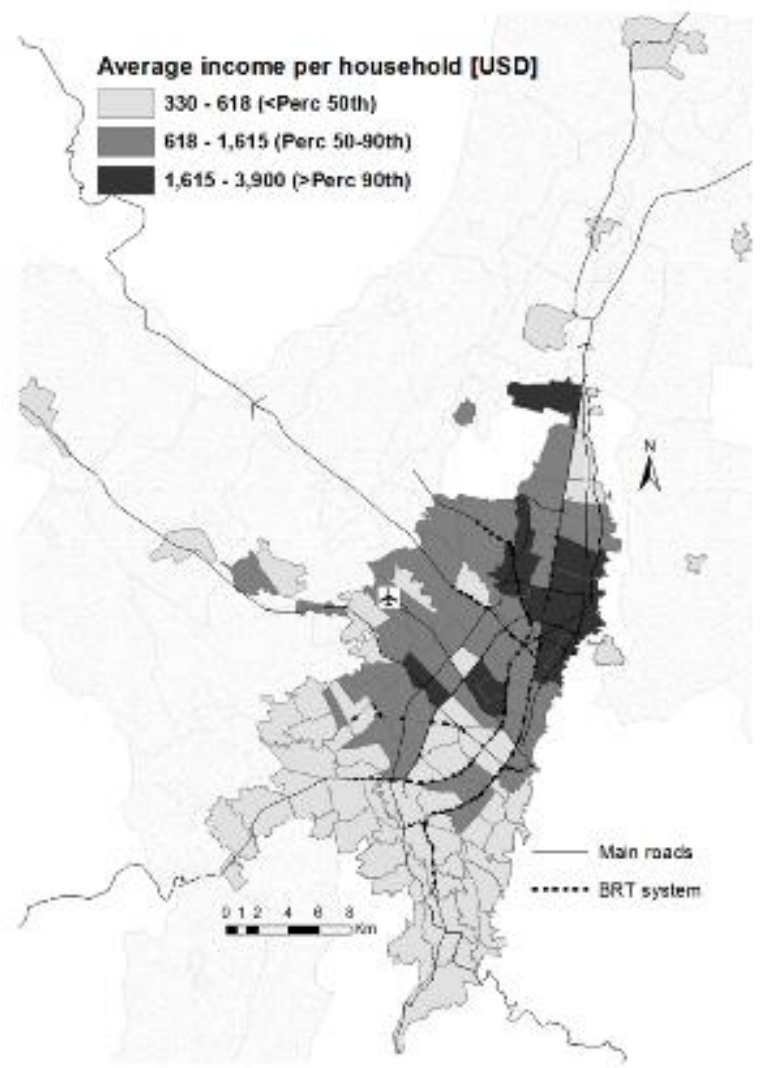

Income level

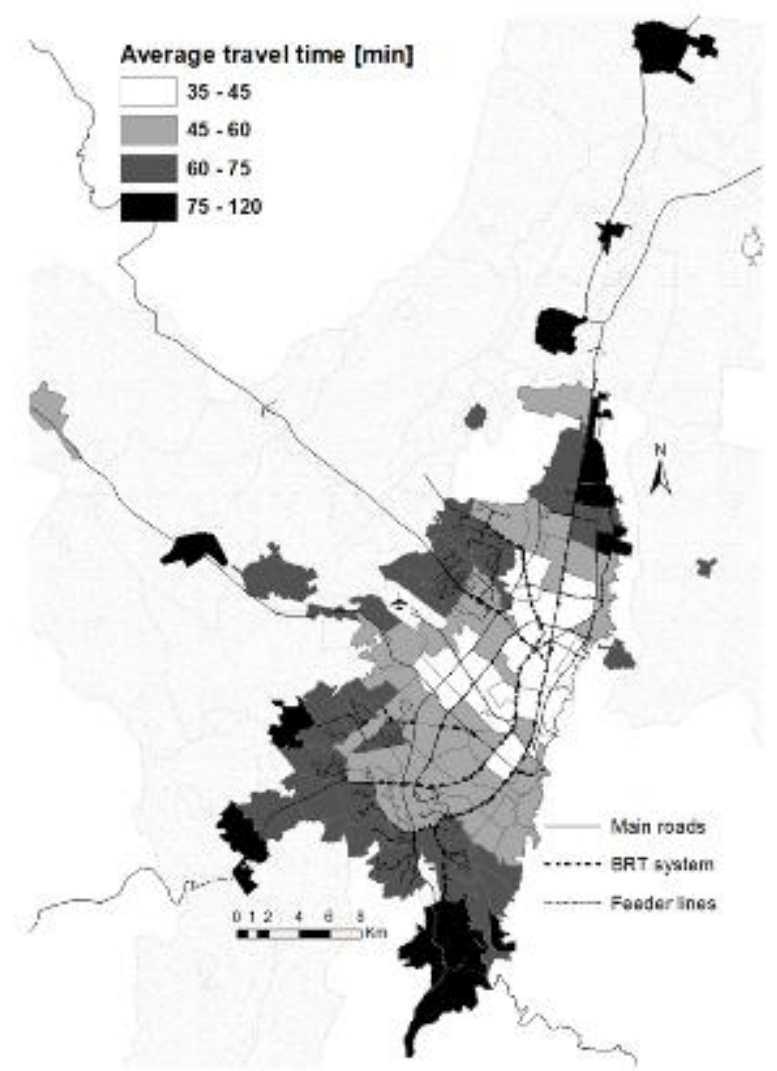

Average travel time (motorized)

Figure 2. Income groups and average travel time by zone

Source: Own elaboration based on data from SDM (2011)

A breakdown of the results by mode of transport shows stark differences between income groups. On average, travel by public transport is 5\% longer in low-income areas, while trips made on the TM are 33\% longer for low-income users in comparison with other income groups. Car ownership is also largely determined by income level. In lowincome zones, there is one car for every five households ( 0.21 vehicles/household), in middle-income zones, one in every two households has at least one vehicle $(0.52$ vehicles/household) and in high-income zones there are 1.3 cars for each household (1.27 vehicles/household). Travel times and vehicles per household were validated using a test of difference of means, finding statistically significant differences at the $95 \%$ confidence level between income groups. These differences are reflected in the average modal split, as in high-income zones more than half of motorized trips devoted to work or study are made via private car $(53 \%)$, while in low-income areas car use barely reaches $15 \%$. This has implications for the analysis of equity in accessibility, as despite the distribution of population of all income levels in the more distant municipalities, income differences allow some inhabitants of the BMR to cover distances by private vehicle, while others are confined to the use of traditional (interurban) public buses. Figure 3 shows the average travel times by mode of transport and income level. 


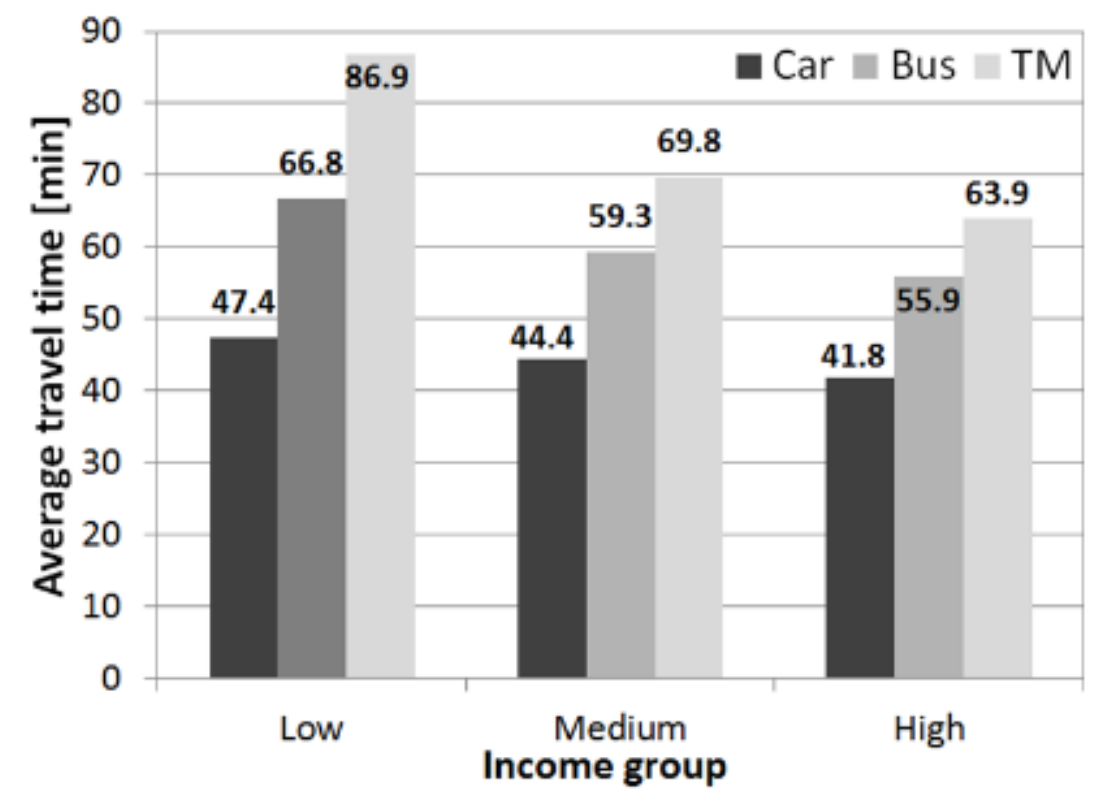

Figure 3. Average travel time to work/study by income group and transport mode

Source: Own elaboration based on data from SDM (2011)

These figures reflect spatial, social and transport-related inequalities in the BMR. The geographical distribution of opportunities in the city, which was consolidated by historic patterns of land occupation and uncoordinated development, benefits higher-income populations. The lack of instruments to influence the socio-spatial distribution of urban and peri-urban spaces (such as metropolitan transport plans, frameworks and regulations for land-use and housing, among others), which can produce a more equitable distribution of access to areas of activity, has generated considerable mobility gaps between high and low income groups. Despite having to cover similar distances as a result of the consolidation of different income groups in municipalities increasingly farther from the city, development of road infrastructure connecting some municipalities and higher access to private vehicles suggest strong differences in accessibility. This also leads to marked differences in mode choice and transport costs involved in mandatory (work/study) travel. Such differences can be identified from a superficial analysis of sociodemographic, transport and land-use variables and supports the perceived need for a detailed analysis focused on equity of accessibility of different social groups to key opportunities in the BMR.

\section{Potential accessibility model}

An effective analysis of how related mandatory accessibility levels distribute in the BMR requires accessibility indicators to be comparable between income groups. We analyzed travel patterns by purpose in the BMR and found that work/study travel patterns are very similar. These in turn differ considerably from the other travel purposes in the city (for shopping, health, recreation, etc.).

The aggregation of employment and education opportunities in accessibility analysis allows for a more complete picture of mandatory travel in the BMR, which governs the main effects and social consequences of transport systems. In a typical day, around 4.7 million mandatory trips are made in the BMR. Of these, $58 \%$ are for work and the rest for study. Trips with other purposes are close to 4.8 million per day but display very different behavior to those of commuting trips. The main effect of combining opportunities is the marginal improvement in accessibility levels in the periphery (in comparison with work-only accessibility), because of a higher supply of education facilities in some peripheral zones. In addition, accessibility on the urban fringe and municipalities tend be 
lower than in central zones due to the spatial distribution of opportunities, mainly employment (see Figure 1). However, these results are correlated with the travel time and travel cost (see Figure 3). We incorporate places of employment and in variables (at the destination) to take into account a measure of the spatial structure of the BMR (Boots and Kanaroglou, 1988).

Assessment of accessibility levels by zone involves the use of a potential accessibility model that incorporates daily trips to work and study by motorized transport modes (car, bus and TM). As argued by Grengs (2010), accessibility by car is greater than accessibility by public transport, a tendency that is often reinforced with distance in the metropolitan context. Our objective is to compare accessibility levels between income groups at a zonal level, as defined in Section 3.2, accounting for the disproportionate dependency of public transport by some social groups.

We calculate accessibility to opportunities using an origin-based measure from each origin zone to all other zones of the BMR by considering the concept of distance weighted by the attributes of a location. In this regard, the influence of a destination zone with fewer and/or more distant opportunities is reduced. The potential accessibility is calculated for all origin zones in the BMR, including the municipalities, which despite larger geographical separation from Bogotá and between each other still show strong inter-municipal travel dynamics and dependency for work and study, partly as a result of the urban primacy of Bogotá (Guzman et al., 2017) and an uneven distribution of opportunities throughout the region. Our calculation of the potential accessibility is a variation of location-based accessibility (Geurs and van Wee, 2004). This is useful for comparing accessibility between different zones (Kwan, 1998). It estimates the accessibility to opportunities from a zone $(I)$ to all other areas $(j)$, considering that fewer or farther opportunities will be less attractive and/or more difficult to reach. Potential accessibility was measured as follows:

$$
A_{i}^{m}=\sum_{j} O_{j} \exp \left(-\beta_{i}^{m} \cdot C_{i j}^{m}\right)
$$

Where $A_{i}^{m}$ is the potential accessibility of the zone $i$ to the transport mode $m, O_{j}$ is the opportunities available at the destination (work and study places); $C_{i j}{ }^{m}$ represents the generalized transport cost by transport mode $m$, i.e. the friction between zones $i$ and $j$ (see equation (2)); and $\beta_{i}^{m}$ is a calibration parameter determined by origin zone and transport mode $m . \beta_{i}^{m}$ parameters were obtained empirically using least median squares (LMS) regressions in order to ensure the estimation of an efficient and unbiased estimator. The dependent variable is the observed accessibility in terms of origin/opportunities and destination zone. The independent variable is the generalized travel cost. For the regressions, observable commuting trips in the origin-destination survey are used as accessed opportunities from zone $i$ to zone $j$, while total opportunities in zone $j$ are calculated from available land-use data as shown in previous figures. In order to obtain the $\beta_{i}^{m}$ calibration parameters per origin and mode, $381 \mathrm{LMS}$ regressions were performed (127 for each transport mode). This parameter was empirically obtained from the regressions to fit the model predictions and the observed distributions of the generalized travel cost functions. Concerning the LMS regressions, each origin had, 88 observations on average. However, only seven zones had fewer than 30 observations and their calibration parameters were imputed from other zones with similar socioeconomic and mobility characteristics. This parameter determines the loss of accessibility in a zone as a result of increasing travel costs.

Additionally, the behavior of the estimators was compared to a previous study (Bocarejo and Oviedo, 2012) in order to test the consistency of the results. Such research suggests that the use of normalized accessibility values allows for comparisons and analysis of relative differences in accessibility between different zones of the city or specific social groups controlled by zone size. Results of equation (1) are normalized in this study using the population of each zone $i$. Normalized results using zone population provide better 
grounds for comparison as even comparatively large absolute numbers of potentially accessible opportunities in relation to the rest of the city may be lower than the actual demand in such zone for activities such as working and studying, which can be an indicator of accessibility deficits.

The generalized travel cost function $\left(C_{i j}{ }^{m}\right)$ is estimated for three transport modes: car, regular public transport (bus) and TM. This differentiation by transport mode seeks to determine the influence of different available transport alternatives on the accessibility of different zones in the BMR. $C_{i j}{ }^{m}$ is an impedance factor, based on money and time, to move between an origin and a destination and is shown as follows:

$$
C_{i j}^{m}=t_{i j}^{m}+{ }^{c_{i j}^{m}} / V O T
$$

Where $t_{i j}{ }^{m}$ represents the travel time between $i$ and $j$ for each transport mode according to the data reported in the sample of the 2011 Mobility Survey; $c_{i j}{ }^{m}$ is the monetary cost associated with each transport mode $m$ between $i$ and $j$; and VOT is the average value of time in the study area (for commuting trips), which was estimated as $69.6 \mathrm{COP} / \mathrm{min}$ (0.037 USD/min) in $2011^{2}$.

For the monetary costs, the following assumptions were taken into account: In the TM system (phases I and II) in 2011 there was a flat rate of COP 1,700 (USD 0.90). In regular buses, the fare was COP 1,450 (USD 0.76), which must be repaid in the case of bus transfers. Finally, for cars an average operating cost per km of COP 426.5 (USD 0.22) was obtained from a land-use-transport model developed by the Universidad de los Andes and the Bogotá Urban Planning Department using VISUM® modelling software. The operating costs reported by the model incorporate the cost of parking at the destination, as well as distance-based costs and recurrent costs such as maintenance, taxes and insurance.

Building on the estimations of potential accessibility we examined how accessibility to work and study is distributed among the population of the BMR. Distribution of accessibility by income groups and transport mode are explored using the income categories defined in Section 3.2. To measure horizontal equity in the BMR, we use Lorenz curves (Delbosc and Currie, 2011b;Lucas et al., 2016) to compare the distribution of accessibility levels by transport mode across the population, employment and study places. These curves are a graphical representation of equity, while the Gini coefficient is an indicator used to determine the overall degree of inequality. The lower the Gini coefficient (closer to zero), the more equitable the distribution of accessibility across the population. A high Gini coefficient (concave curve) implies an unequal distribution.

These results, however, do not allow for comparisons across income groups in their aggregate form. Particularly considering differences in the modal split between such groups. To measure vertical equity, the accessibility levels to work/study were compared across the population. Accessibility levels are plotted against the percentile of the population in order to examine inequalities between income groups.

\section{Results}

Results of potential accessibility require careful interpretation: numbers of "achievable" opportunities are weighted according to the travel costs from the trip origin. Readability of the results can be improved by showing separately the effect of the spatial distribution of activities and time or travel costs on the accessibility indicator (Figures 1 and 2, right).

The parameters of $\beta_{i}^{m}$ in equation (1) are overall significant for each zone in the BMR. The car mode of transport had 126 parameters significant at the $99 \%$ confidence level. Public transport (regular buses) shows 118 significant coefficients at 99\%, 4 at $95 \%, 3$

\footnotetext{
2 The value of time is based on own studies (unpublished) of the University of the Andes and Bogotá Urban Planning Office Department.
} 
at $90 \%$, and for only two zones were the calibration parameters not significantly different from zero. Finally, in TM, 122 factors had a significance of $99 \%, 1$ at $90 \%$ and four zones had parameters not significantly different from zero. In cases where the parameters showed insufficient statistical significance, the coefficients from a neighboring zone with similar socioeconomic and mobility characteristics were assigned.

As specified in the previous section, normalization of the accessibility levels by transport mode for each zone of the study area (UPZ and municipalities) was conducted by dividing the accessibility value of each zone by the zone's population. These normalized values allow for a focus on the relative differences between zones rather than the absolute values, which are largely meaningless except in a relative sense (Handy, 1994). Results are shown in Figure 4. Per capita accessibility levels contrast with population distribution and education and employment opportunities in the BMR. Public transport accessibility per capita suggests the greater benefits of this system in and around the city center and certain zones in the periphery. The spatial distribution of opportunities is an explanatory factor in the results of accessibility for inhabitants using private vehicles (cars). Availability of cars is more closely related to household income level and costs related to the distances traveled, which leads to longer trips originating in high-income zones and thus greater accessibility.

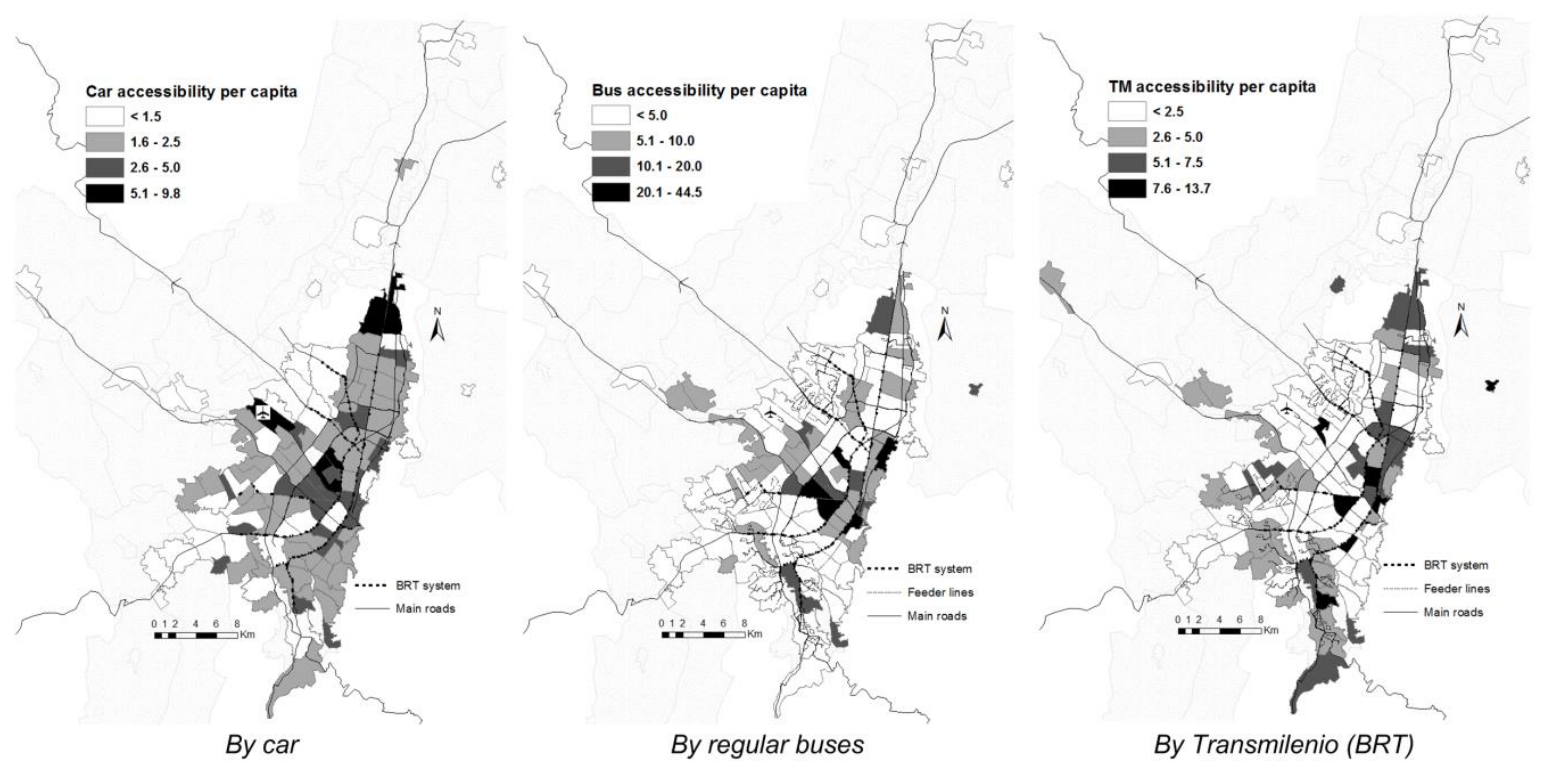

Figure 4. Potential accessibility per capita

Source: Own elaboration.

Grouping accessibility results by income group reveal access inequalities throughout the BMR. As shown in Table 1, average accessibility levels (1.91) per inhabitant by car in the low-income group (column 1) are the lowest among the three income categories. Individual accessibility per capita by car in the low-income group is $88 \%$ of the average per capita accessibility of the BMR (2.16). By contrast, results in the medium and high income groups of average per capita accessibility by car are considerably higher, being respectively 1.10 and 1.16 times higher than the BMR's average accessibility by this mode (Table 1). In regular buses, these differences increase: the low-income group has an accessibility that is only $84 \%$ of the region's average accessibility per inhabitant (column 2), while the accessibility results for the medium and the high-income group are 1.11 and 1.31 times higher than the average, respectively. This implies that the wealthiest groups enjoy $11 \%$ and $31 \%$ more accessibility than the average. In TM (column 3), these indicators are 0.95, 1.04 and 1.12 times the BMR average for the low, middle and high-income groups, respectively. These indicators suggest the negative 
effect of the spatial distribution of opportunities and transport within the city on equity, particularly in relation to the low-income population.

Table 1. Average accessibility levels per capita and income groups

\begin{tabular}{|l|c|c|c|}
\hline \multicolumn{1}{|c|}{ Income group } & Car (1) & Bus (2) & TM (3) \\
\hline Low & 1.91 & 5.20 & 3.05 \\
\hline Medium & 2.38 & 6.87 & 3.35 \\
\hline High & 2.50 & 8.07 & 3.63 \\
\hline Total BMR & $\mathbf{2 . 1 6}$ & $\mathbf{6 . 1 7}$ & $\mathbf{3 . 2 2}$ \\
\hline
\end{tabular}

Source: Own elaboration.

\subsection{Horizontal equity across the BMR population}

To measure relative mode-based accessibility levels of the population in each zone, we estimate Gini coefficients throughout the BMR. Since this indicator only considers income, we made adjustments to reflect potential accessibility. This is a standard measure of overall inequality, based on Lorenz Curves (Brown, 1994), where 0 corresponds to perfect equality (i.e. everyone has the same accessibility levels) and 1 corresponds to perfect inequality (i.e. one individual has all the accessibility, while everyone else has zero accessibility). This coefficient was 0.326 and 0.348 for the accessibilities by car and public transport, respectively.

Figure 5 (left) shows the Lorenz curve where accessibility levels were compared to the residential population in each zone. This means that if accessibility is interpreted as the amount of opportunities, $50 \%$ of the BMR's population shared only $32 \%$ of the opportunities offered by the city (for car users). In the same vein, for public transport users, $60 \%$ of population shared $40 \%$ of the opportunities. Conversely, $40 \%$ of the BMR's population shared $60 \%$ of the city opportunities.

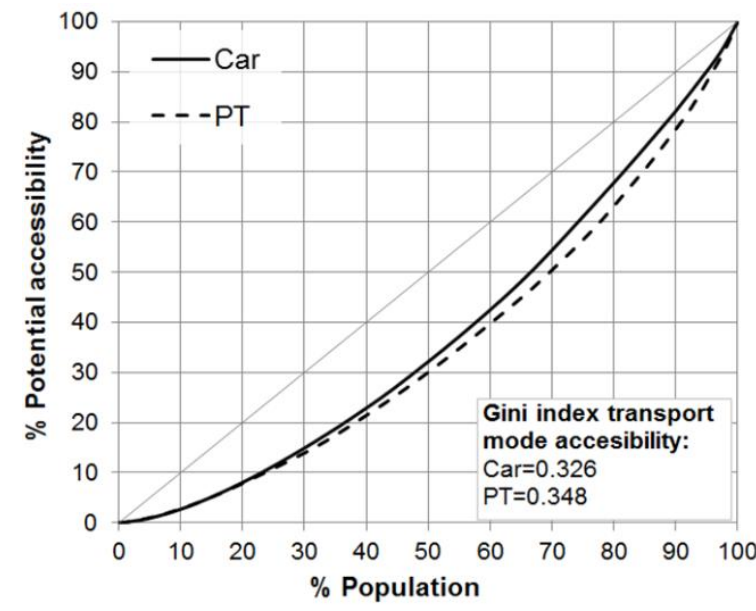

Accessibility to population

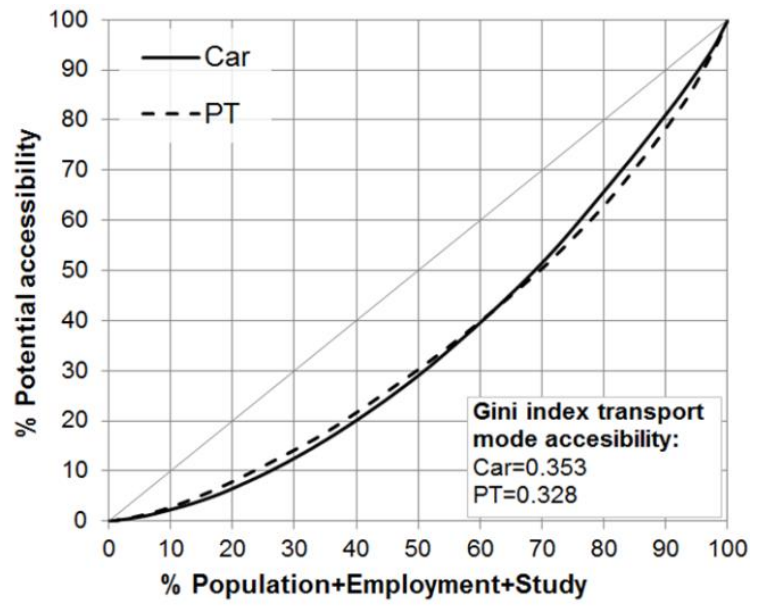

Accessibility to

population+workplaces+studyplaces

Figure 5. Lorenz curves of population, employment and study places

Source: Own elaboration

This accessibility calculation was made taking into account origins (location of the population) and destinations (jobs and study places). As the purpose of the transport system is to serve both origins and destinations, a second Lorenz curve was built comparing origin-based accessibility levels by transport mode to a combination of residential, employment and study places (Figure 5, right). By doing this, the car accessibility distribution was slightly less equitable ( $\mathrm{Gini}=0.353,8.4 \%$ more inequitable), while some improvement in public transport accessibility distribution is observed 
(Gini $=0.328,5.8 \%$ more equitable). This is due to the high supply of public transport in the zones where the greatest concentration of employment and study facilities are found.

Gini coefficients for the potential accessibility are powerful indicators of the disparities between the previously defined socio-demographic groups. Overall, these results suggest a relatively good horizontal equity, as higher accessibility levels are available to a large share of the population. These results are affected when considering competitive factors and differences between requirements (education skills and training) to participate in different employment and education opportunities. In this regard, particularly in low-income groups where the education levels and literacy rates are lower, a drastic reduction of this accessibility is expected in practice.

\subsection{Vertical equity across the income groups}

The aim of these analyses is to identify differences in accessibility levels throughout the BMR according to income groups. The accessibility differences observed between transport modes are associated with coverage levels and the availability of different transport systems, whose planning tends to benefit high-income zones, as well zones with large activity (Graham and Marvin, 2001;Oviedo and Dávila, 2016). We identify disadvantaged groups, seeking to support the development of actions that betteraccommodate their needs (e.g. implement targeted public transport subsidies for specific segments of the population).

We use the proposed methodology to quantify and compare the potential accessibility of the three previously defined income groups to employment and study places in the BMR, taking into account time and financial transport costs. Figure 6 shows the vertical equity results, where accessibility levels are included on the vertical axis, and the percentiles of the population on the horizontal axis, thus comparing the three income groups by transport mode.

Results show that for car accessibility, the high-income group has an evenly distribution (i.e., looking at the 50th percentile of the population, half of the population belonging to this group, experience approximately the same average accessibility levels in the BMR). The line proceeds in a straight rise, with a relatively constant slope. This means that half of the high-income zones experience lower levels of car accessibility, while the other half enjoy higher -than- average accessibility levels. This distribution contrasts with the curves of the other income groups, where more inequitable distributions are obtained. In these cases, about $70 \%$ of the population of these two income group experience higher accessibility levels than the average. However, it is important to remember that in these zones car ownership is between 6.0 and 2.5 times lower than in high-income zones.

Regarding bus accessibility, greater inequalities were found in high-income zones than in low-income and medium-income zones, as can be seen by the contrasting shapes of the three lines. Up to the $60^{\text {th }}$ percentile, the shapes of the three lines are very similar, suggesting little difference between income groups. About half of the population in the BMR experiences moderate advantages in access to work/study opportunities; otherwise, accessibility is fairly comparable across the income groups throughout the rest of the population percentiles. This is because there are zones (UPZ) in the city center, where there are comparatively few residents, but that have many jobs and study places in addition to good public transport. 

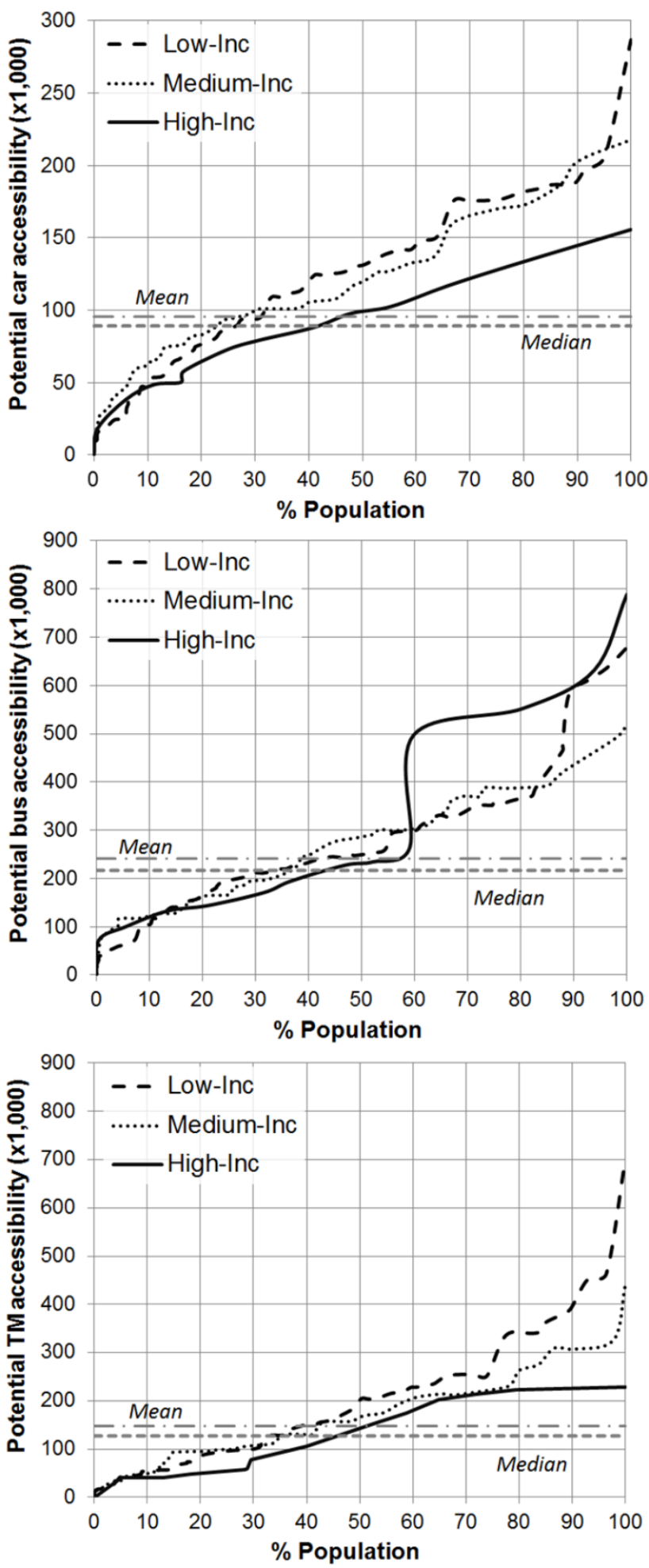

Figure 6. Accessibility to work/study by transport mode and income group

Source: Own elaboration

A similar trend is observed in TM results, which is reflected by the contrast between the shapes of the curves. For low and medium income groups, the curve proceeds in and (almost) straight rise, forming a nearly perfect diagonal from the origin. The slope of these curves increases significantly at the value of approximately 80 on the $x$-axis. This means that about $20 \%$ of low and medium income groups experience extremely high accessibility (between 2 and 5 times the average). For the remaining $80 \%$ of low and 
middle income groups accessibility in TM is evenly distributed: about half of the population experience high accessibility, and about half experience low accessibility. This is explained by the low coverage of the TM system compared to traditional buses and private vehicles, leading to several zones having no access to this service. Another element to highlight from these results is the low accessibility levels of TM in high-income zones, compared to the others. The location of most feeder routes in low-income zones, and trunk corridors mainly in medium-income zones can explain this.

Results indicate that under the current conditions of opportunity distribution and distribution of the transport system, most of the low-income population is significantly disadvantaged compared to wealthier groups. This can be potentially addressed through policies that alleviate travel costs for the poor and low-income groups such as targeted subsidies, and land-use incentives for the redistribution of employment and urban education centers.

\subsection{Equity index}

Finally, in order to better understand equity differences we compared the proportion of accessibility and the proportion of population in each zone. That is, for each zone, we want to know what degree of accessibility is experienced in relation to the total accessibility in the city and the share of the total population in each origin zone. We estimated a ratio of the population and accessibility for each zone in the BMR. Results of this indicator allow us to pinpoint zones with higher potential accessibility than residents and vice versa. In other words, in zones where this indicator is less than 1 an imbalance between population and accessibility is implied (i.e. there are more residents than potentially accessible opportunities). In contrast, zones with values greater than 1 have more accessibility per inhabitant (see Figure 7). This indicator is calculated under the assumption that the entire active population in the zone should at least be able to access one opportunity of either work or study.

These results show differences in the distribution of accessibility in a cumulative fashion according to the population. For the car, we found zones at the peripheries of the city had high accessibility levels according to the population. This can be explained by a relatively low resident population (between 1,000 and 4,000 people) in comparison with average population per zone in the BMR, which is about 60,000.

Public transport shows the most interesting results. When comparing proportional accessibility results with income groups (Figure 2) it is possible to identify a greater imbalance between accessibility and population in low-income zones. This is reflected in average travel time and the location of work and study opportunities. Most of the population does not live where the majority of activities are located. The BMR, like other major metropolises in Colombia and Latin America, tends to maintain a centralized distribution of economic opportunities, which increases the land and housing prices in these high-activity areas and pushes the poor toward more affordable, housing, often informal, at the urban peripheries or suburban areas. 


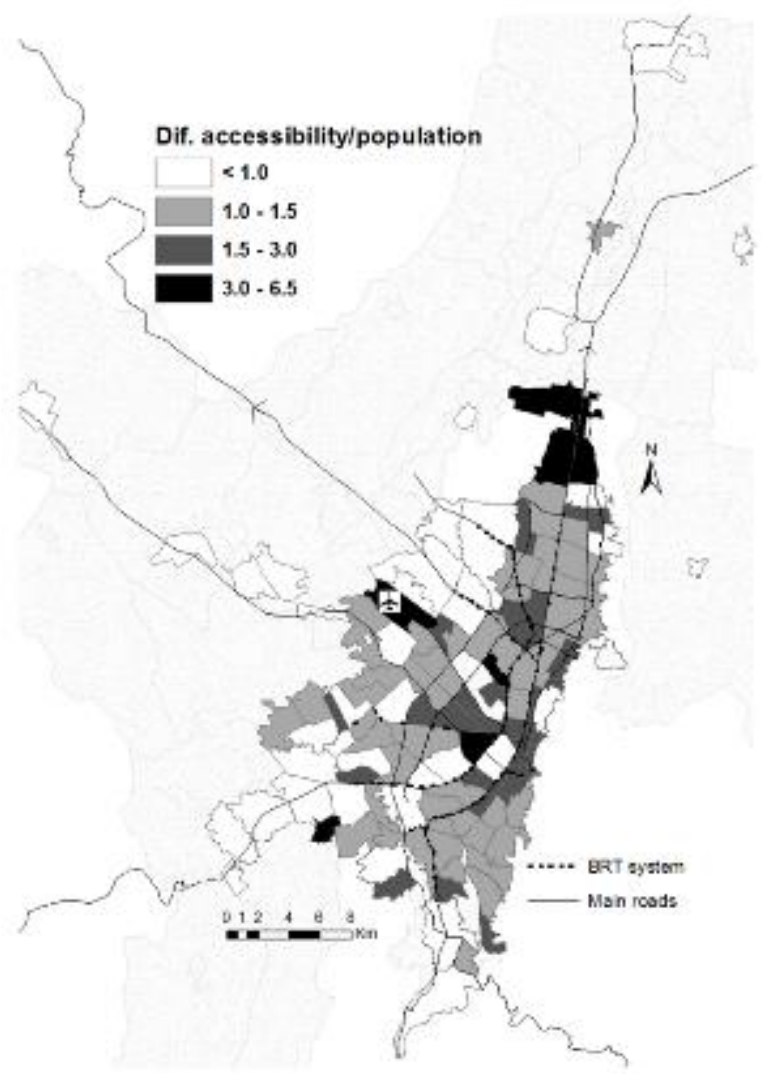

By car

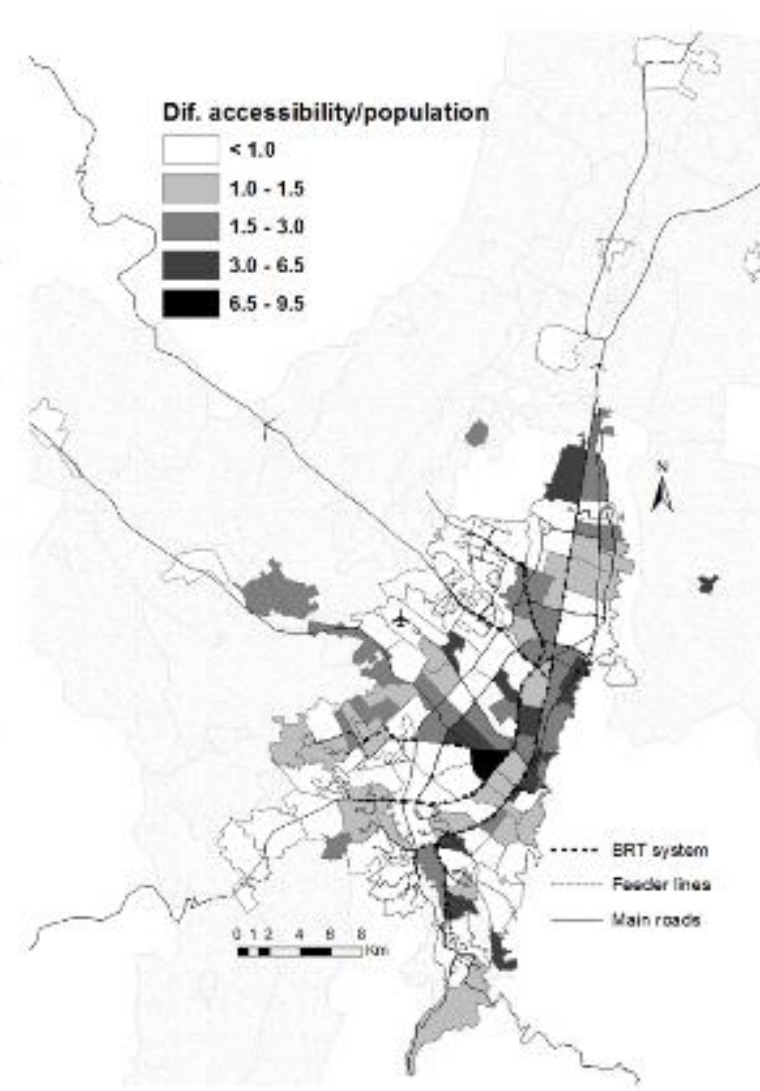

By public transport

Figure 7. Accessibility and population differences according to the transport mode

Source: Own elaboration

\section{Discussion and conclusion}

Results regarding accessibility in the BMR help to identify social, spatial, and transportrelated inequalities that were initially suggested by sociodemographic and economic figures. The spatial distribution of opportunities and social groups are significant explanatory factors in the reproduction of disparities between users of the same transport mode at different locations. Such an influence is reflected not only in the parameters of the functions of impedance used for the estimation of accessibility by income groups, but also in the variables of expenditure themselves, which evidence the ability of specific modes of motorized transport, like collective and mass public transport and cars, to increase the accessibility of their users, particularly in the case of low-income users. The agglomeration of attractiveness and wealth in specific areas of the city reinforces the distribution of infrastructure and public transport services in areas already hosting the majority of economic and education-related opportunities, which is reflected by higher results in all indicators for central zones.

The use of Lorenz curves and Gini coefficients for the analysis of disparities in accessibility has an added value in cases with marked concentrations of opportunities and socio-spatial population segregation, as in many cities in the Global South. They allow us to understand the effects of differences in supply coverage, social, economic, and geographical elements underpinning travel choice in the distribution of accessibility throughout urban and metropolitan areas. In this regard, these indicators can serve as supporting evidence of the need for localized interventions and integrated planning that add value to mainstream accessibility analysis and approaches in spatial and transport planning. Our results also bear relevance for the critique of the criteria for investment prioritization and interventions to infrastructure and transport systems, even in high- 
income areas. The marked differences in accessibility between income groups allows us to identify areas that are better-off and worst-off in terms of access to employment and education, which can be used as an additional criterion for decision-making. Although it is true that higher-income areas have, in general, better average access to these opportunities, as shown by the results, this may be related to specific areas having disproportionate potential access that is not being fully exploited by the city-region.

The methodology used in this study can be replicated in other contexts where information on employment and education, as well as sufficient data on travel costs, are available. Information from travel demand models for the estimation of travel costs are another potential source of information. Furthermore, although the study was developed in the context of the BMR, the methodology is transferrable to the study of other cases. In terms of interpretation, we suggest that the socio-spatial structure of the BMR, which has governed most of the insights contained in this paper, is one of the strongest features accounting for the social and spatial segregation.

To conclude, this research adds empirical and methodological elements to current debates and approaches of the analysis of accessibility in Latin America from three perspectives. First, by incorporating the metropolitan-scale in the analysis, results evidenced the consolidation of social and spatial inequalities that arise from a decentralized planning approach to transport and land-use in a spatially and functionally conurbated urban area, such as the BMR. Second, the incorporation of metrics of inequality, such as Gini coefficients and Lorenz curves, to the analysis of accessibility were proven to be powerful tools for visualizing and identifying differences in access to employment and education, which can be used in practice to better-communicate the social consequences of urban transport to decision-makers. Third, the differentiation of the results by transport mode and income group can help provide insights in relation to the demand-supply interactions of such modes, allowing better identification of priorities for intervention, aimed at both improving coverage and access and redistributing current levels of accessibility (i.e., congestion tolls and/or circulation restrictions).

\section{Acknowledgements}

We would like to express our gratitude to the Bogotá Urban Planning Department (www.sdp.gov.co), for the funding and general support given for the development of this study.

\section{References}

Acosta, P., 2010. The Bogota - Sabana Region: The political economy behind the struggle to implement a sustainable urban development model, Universidad del Rosario.

Alam, B., 2009. Transit Accessibility to Jobs and Employment Prospects of Welfare Recipients Without Cars, Transportation Research Record: Journal of the Transportation Research Board 2110, 78-86.

Ben-Akiva, M. E., Dong, X., Bowman, J. L., Walker, J. L., 2006. Moving from trip-based to activity-based measures of accessibility, Transportation Research Part A: Policy and Practice 40(2), 163-180.

Bocarejo, J. P., Oviedo, D. R., 2012. Transport accessibility and social inequities: a tool for identification of mobility needs and evaluation of transport investments, Journal of Transport Geography 24, 142-154.

Bocarejo, J. P., Portilla, I. J., Velásquez, J. M., Cruz, M., Peña, A., Oviedo, D., 2014. An innovative transit system and its impact on low income users: the case of the Metrocable in Medellín, Journal of Transport Geography 39, 49-61. 
Boots, B. N., Kanaroglou, P. S., 1988. Incorporating the effects of spatial structure in discrete choice models of migration, Journal of Regional Science 28(4), 495-510.

Brown, M., 1994. Using Gini-style indices to evaluate the spatial patterns of health practitioners; theoretical considerations and an application based on the Alberta data, Social Science and Medicine 38(9), 1243-1256.

CCB. Cámara de Comercio de Bogotá. (2015). Documento maestro de diagnóstico sobre la situación y retos de Bogotá Región para pre candidatos a la Alcaldía Mayor de Bogotá y a la Gobernación de Cundinamarca. Bogotá, Colombia

Dalvi, M. Q., Martin, K. M., 1976. The measurement of accessibility: Some preliminary results, Transportation 5(1), 17-42.

DANE. (2013a). National Department of Statistics. Encuesta Nacional de Calidad de Vida.

DANE. (2013b). National Department of Statistics. Gran Encuesta Integrada de Hogares - GEIH

Deakin, E., 2007. Equity and Environmental Justice in Sustainable Transportation: Toward A Research Agenda, UC Berkeley: University of California Transportation Center.

Delbosc, A., Currie, G., 2011a. The spatial context of transport disadvantage, social exclusion and well-being, Journal of Transport Geography 19(6), 1130-1137.

Delbosc, A., Currie, G., 2011b. Using Lorenz curves to assess public transport equity, Journal of Transport Geography 19(6), 1252-1259.

Delmelle, E. C., Casas, I., 2012. Evaluating the spatial equity of bus rapid transit-based accessibility patterns in a developing country: The case of Cali, Colombia, Transport Policy 20, 36-46.

Escobar, D., Garcia, F., 2012. Territorial Accessibility Analysis as a Key Variable for Diagnosis of Urban Mobility: A Case Study Manizales (Colombia), Procedia - Social and Behavioral Sciences 48, 1385-1394.

Geurs, K. T., Ritsema van Eck, J. R., 2001. Accessibility measures: review and applications, Netherlands Environmental Assessment Agency.

Geurs, K. T., van Wee, B., 2004. Accessibility evaluation of land-use and transport strategies: review and research directions, Journal of Transport Geography 12(2), 127140.

Gilbert, A., 2008. Bus Rapid Transit: Is Transmilenio a Miracle Cure? Transport Reviews 28(4), 439-467.

Graham, S., Marvin, S. (Eds), , 2001. Splintering Urbanism: Networked Infrastructures, Technological Mobilities and the Urban Condition. London: Routledge.

Grengs, J., 2015. Nonwork Accessibility as a Social Equity Indicator, International Journal of Sustainable Transportation 9(1), 1-14.

Grengs, J., 2010. Job accessibility and the modal mismatch in Detroit, Journal of Transport Geography 18(1), 42-54.

Grindlay, A. L., Jaramillo, C., Lizárraga, C., 2012. Spatial disparity in transport social needs and public transport provision in Santiago de Cali (Colombia), Journal of Transport Geography 24, 340-357.

Guzman, L. A., Moncada, C. A., Ochoa, A., 2016. The peak problem and fare reduction effect in the Transmilenio system in Bogota, Colombia. World Conference on Transport Research - WCTR 2016 Shanghai. 
Guzman, L. A., Oviedo, D., Bocarejo, J. P., 2017. City profile: The Bogotá Metropolitan Area that never was, Cities 60, Part A, 202-215.

Handy, S., 1994. Regional Versus Local Accessibility: Implications for Nonwork Travel, Transportation Research Record 1400, 58-66.

Hansen, W. G., 1959. How Accessibility Shapes Land Use, Journal of the American Planning Association 25(2), 73-76.

Hess, D. B., 2005. Access to Employment for Adults in Poverty in the Buffalo-Niagara Region, Urban Studies 42(7), 1177-1200.

Hidalgo, D., Sandoval, E., 2004. TransMilenio: A High Capacity - Low Cost Bus Rapid Transit System Developed for Bogotá, Colombia. American Society of Civil Engineers, pp. 37-49.

Hine, J., 2009. The provision of home to school transport in Northern Ireland, Research in Transportation Economics 25(1), 29-38.

Jones, P., Lucas, K., 2012. The social consequences of transport decision-making: clarifying concepts, synthesising knowledge and assessing implications, Journal of Transport Geography 21, 4-16.

Karlström, A., Franklin, J. P., 2009. Behavioral adjustments and equity effects of congestion pricing: Analysis of morning commutes during the Stockholm Trial, Transportation Research Part A 43(3), 283-296.

Kawabata, M., 2003. Job Access and Employment among Low-Skilled Autoless Workers in US Metropolitan Areas, Environment and Planning A 35(9), 1651-1668.

Keeling, D. J., 2008a. Latin America's Transportation Conundrum, Journal of Latin American Geography 7(2), 133-154.

Keeling, D. J., 2008b. Transportation geography - new regional mobilities, Progress in Human Geography 32(2), 275-283.

Korsu, E., Wenglenski, S., 2010. Job Accessibility, Residential Segregation and Risk of Long-term Unemployment in the Paris Region, Urban Studies 47(11), 2279-2324.

Kwan, M., 1998. Space-Time and Integral Measures of Individual Accessibility: A Comparative Analysis Using a Point-based Framework, Geographical Analysis 30(3), 191-216.

Levinson, D. M., 1998. Accessibility and the Journey to Work, Journal of Transport Geography 6(1), 11-21.

Litman, T., 2014. Evaluating Transportation Equity: Guidance for Incorporating Distributional Impacts in Transportation, Victoria Transport Policy Institute.

Lucas, K., 2012. Transport and social exclusion: Where are we now? Transport Policy 20, 105-113.

Lucas, K., Jones, P., 2012. Social impacts and equity issues in transport: an introduction, Journal of Transport Geography 21, 1-3.

Lucas, K., van Wee, B., Maat, K., 2016. A method to evaluate equitable accessibility: combining ethical theories and accessibility-based approaches, Transportation 43(3), 473-490.

Manderscheid, K., 2009. Unequal Mobilities. In: Ohnmacht, T., Maksim, H., Bergman, M. M. (Eds), Mobilities and Inequality: Ashgate Publishing Ltd., pp. 27-50.

Munoz-Raskin, R., 2010. Walking accessibility to bus rapid transit: Does it affect property values? The case of Bogotá, Colombia, Transport Policy 17(2), 72-84. 
Oviedo, D., Dávila, J. D., 2016. Transport, urban development and the peripheral poor in Colombia - Placing splintering urbanism in the context of transport networks, Journal of Transport Geography 51, 180-192.

Preston, J., Rajé, F., 2007. Accessibility, mobility and transport-related social exclusion, Journal of Transport Geography 15(3), 151-160.

Sanchez, T. W., Stolz, R., Ma, J. S., 2003. Moving to Equity: Addressing Inequitable Effects of Transportation Policies on Minorities, The Civil Rights Project at Harvard University, Cambridge, MA.

Scholl, L., Bouillon, C., Oviedo, D., Corsetto, L., Jansson, M., 2016. Urban Transport and Poverty: Mobility and Accessibility Effects of IDB-supported BRT Systems in Cali and Lima, Inter-American Development Bank.

SDM, 2011. Bogotá Mobility Survey 2011, Secretaria Distrital de Movilidad de Bogotá.

Thibert, J., Osorio, G. A., 2014. Urban Segregation and Metropolitics in Latin America: The Case of Bogotá, Colombia, International Journal of Urban and Regional Research 38(4), 1319-1343.

Titheridge, H., Christie, N., Mackett, R., Oviedo, D., Ye, R., 2014. Transport and Poverty. A review of the evidence, Report for the Joseph Rowntree Foundation.

van Wee, B., 2016. Accessible accessibility research challenges, Journal of Transport Geography 51, 9-16.

van Wee, B., Geurs, K., Chorus, C., 2013. Information, communication, travel behavior and accessibility, Journal of Transport and Land Use 6(3), 1-16.

van Wee, B., Handy, S., 2016. Key research themes on urban space, scale, and sustainable urban mobility, International Journal of Sustainable Transportation 10(1), 1824.

Wachs, M., 2010. Transportation Policy, Poverty, and Sustainability, Transportation Research Record: Journal of the Transportation Research Board 2163, 5-12.

Wu, B. M., Hine, J. P., 2003. A PTAL approach to measuring changes in bus service accessibility, Transport Policy 10(4), 307-320. 